Reliable Computing 2 (3) (1996), pp. 219-228

\title{
Fast error estimates for indirect measurements: applications to pavement engineering
}

\author{
Carlos Ferregut, Soheil Nazarian, Krishnamohan Vennalaganti, \\ Ching-Chuan Chang, and Vladik Kremonich
}

In many real-life situations, we have the following problem: we want $t o$ know the value of some characteristic $y$ that is difficult to measure directly (e.g., lifetime of a pavement, efficiency of an engine, etc.). To estimate $y$, we must know the relationship between $y$ and some directly measurable physical quantities $x_{1}, \ldots, x_{n}$. From this relationship, we extract an algorithm $f$ that allows us, given $x_{i}$, to compute $y: y=f\left(x_{1}, \ldots, x_{n}\right)$. So, we measture $x_{i}$, apply an algorithm $f$, and get the desired estimate.

Existing algorithms for error estimate (interval mathematics, Monte-Carho methods, numerical differentiation, etc.) require computation time that is several times larger than the time necessary to compute $y=f\left(x_{1}, \ldots, x_{n}\right)$. So, if an algorithm $f$ is already time-consuming, error estimates will take tox long.

In many cases, this algorithm $f$ consists of two parts: first, we use $x_{i}$ to determine the parameters $z_{k}$ of a mudel that describes the measured object, and second, we use these parameters to estimate $y$. The most time-consuming part is finding $z_{k}$ i this is done by solving a system of non-linear equations; usually least squares method is used.

We show that for such $f$, one can estimate errors repeating this time-consuming part of $f$ only once. So, we can compute both $y$ and an error estimate for $y$ with practically no increase in total computation time. As an example of this methodology, we give pavement lifetime estimates.

\section{Быстрое оценивание погрешностей при непрямых измерениях: приложения к проектированию мостовых}

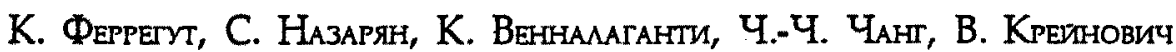

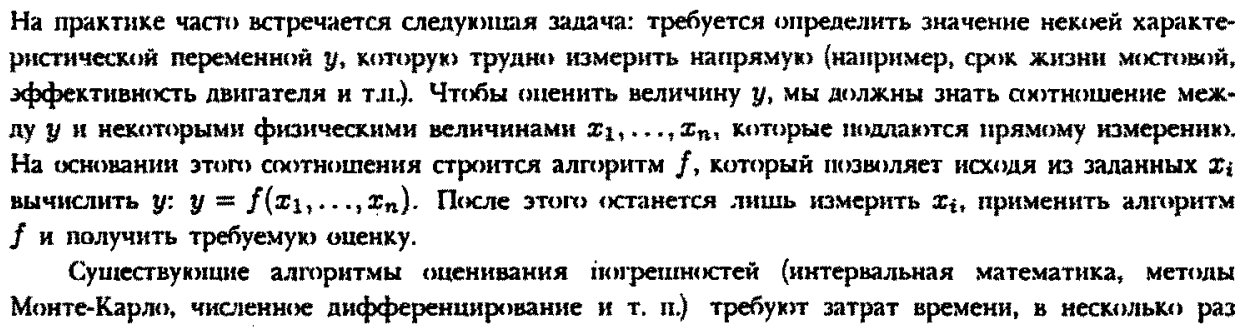

(C) C. Ferregut, S. Nazarian, K. Vennalaganti, C.-C. Chang, V. Kreinovich, 1996

This work was supported by NSF grants No. CDA-9015006 and IRI-92-11-662, NASA Research Grant No. 9482, and a grant from the Materials Research Institute. We want to thank the United States Army Corps of Engineers, Waterways Experiment Station (USAE, WES), Vicksburg, MS, for supplying us with the Layered Elastic Programs, WELSEA and WESDEF. We are also greatly thankful to all the participants of the International Conference on Ni1merical Analysis with Autumatic Result Verification, Lafayette, LA, Febriary-March 1993, especially to Drs. S. Markov and $W$. Walster, for valuable discussions. 
иревыпшамиих те, которые нужны лля иычисления $y=f\left(x_{1}, \ldots, x_{n}\right)$. Пиэтьму, если алтритм $f, k$ без того рабитает медленно, оиенивание иогреинистей мижет занять нетрнемлемо болыпке иремя.

Во многих случаях алиритм $f$ систит из лиух частей: сначала на иснове $x_{i}$ өпределимтся

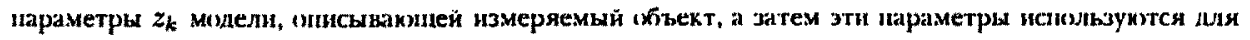
oиекки $y$. Нанболыних временных затрат требует шлучение значений $z_{k}$, для чего нетбхиимо решить систему нелинейных уравнений (Как правили, применяется метод наименыших киалатов).

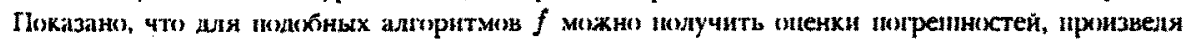
эту трудемхую чась иычислений тольхо одия раз. Таким иқразом, мы можем вычислить как

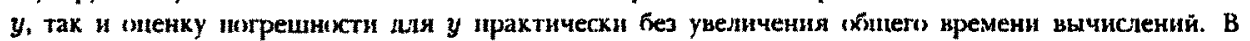

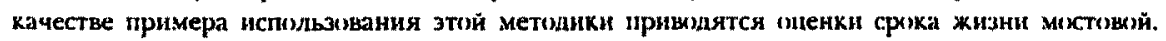

\section{Formulation of a general problem: error estimation for indirect measurements}

Indirect measurements: a real-life situation. In this paper, we will consider the following engineering problem:

We want: to know the value of some physical quantity $y$.

Problem: It is difficult (or even impossible) to measure $y$ directly (examples of such quantities are lifetime of a pavement, efficiency of an engine, etc.).

Solution: To estimate $y$, we must know the relationship between $y$ and some directly measurable physical quantities $x_{1}, \ldots, x_{n}$. From this relationship, we extract an algorithm $f$ that allows us, given $x_{i}$, to compute $y: y=f\left(x_{1}, \ldots, x_{n}\right)$. So, we measure $x_{i}$, apply an algorithm $f$ to the measurement results $\tilde{x}_{i}$, and get the desired estimate $\tilde{y}=f\left(\tilde{x}_{1}, \tilde{x}_{2}, \ldots, \tilde{x}_{n}\right)$. This procedure is called an indirect measurement of $y$.

Warning: This $f$ is rarely an explicit analytical expression; usually, it is a complicated algorithm.

Remaining problem: What is the precision of the resulting value $\tilde{y}$ ?

What do we know about the errors of measuring $x_{i}$ ? The error of an indirect measurement is caused by the errors with which we know $x_{i}$. So, to estimate the precision of $y$, let us find out what we know about these precisions.

For each measuring device, its supplier must provide some information about its precision (else, if no precision is guaranteed, this measuring device is of no use). There are two main ways to describe such a precision (see, e.g., [4]):

1) In many cases, we know only a characteristic of the total error $\Delta x_{i}=\bar{x}_{i}-x_{i}$. Namely, the supplier provides a number $\Delta_{i}$ such that the error never exceeds $\Delta_{i}\left(\left|\Delta x_{i}\right| \leq \Delta_{i}\right)$; in other words, the actual value $x_{i}$ belongs to an interval $\left[\tilde{x}_{i}-\varepsilon, \tilde{x}_{i}+\varepsilon\right]$.

This number $\Delta_{i}$ can depend on $\tilde{x}_{i}$. For example, a supplier can provide us with a relative error $\delta_{i}$, meaning that $\left|\Delta x_{i}\right| \leq \delta_{i} \bar{x}_{i}$ (so, in this case, $\Delta_{i}=\delta_{i} \bar{x}_{i}$ ).

2) Measurement error $\Delta x$ is random. Therefore, we can represent it as a sum of two components: the mathematical expectation $E(\Delta x)$ that is called a systematic error (denoted by $\Delta x_{i}^{s}$ ), and the difference $\Delta x-E(\Delta x)$ that is called a random error (and denoted by $\Delta x_{i}^{r}$ ). For some measuring devices, we know the characteristics of the both components. Namely, the supplier provides a value $\Delta_{i}^{s}$ such that $\left|\Delta x_{i}^{s}\right| \leq \Delta_{i}^{s}$, and a value $\sigma_{i}$ such that the standard deviation $\sigma\left(\Delta x_{i}\right)$ does not exceed $\sigma_{i}$. 
These two characteristics can also depend on $\tilde{x}_{i}$. For example, they can be given as relative errors $\delta_{i}^{s}$ and $s_{i}$. In this case, $\Delta_{i}^{s}=\bar{x}_{i} \delta_{i}^{s}$ and $\sigma_{i}=\bar{x}_{i} s_{i}$.

Systematic errors are usually negligible. To improve the quality of a measuring device, it makes sense to calibrate it first. By this we mean that before we release this device, we compare its results with the results of a more precise device, find an average error, and compensate for it. For calibrated devices, the systematic error is negligible, so we can assume that the total error coincides with its random component: After the calibration, we also have enough data to estimate the standard deviation $\sigma\left(x_{i}\right)$. So, we can assume that $\sigma\left(x_{i}\right)$ is known.

In view of this, in this paper, we will consider only two cases:

1) we known an interval for an error;

2) the error has 0 average, we know its standard deviation $\sigma\left(x_{i}\right)$, and the errors of different measurements are independent random variables.

\section{How are errors estimated now?}

The main methodologies for estimating the error of $y$ are: numerical differentiation, MonteCarlo method (for detailed description of these methods, see, e.g., [1, 2, 5, 13-15]), and interval mathematics (see, e.g., [11]). Let us briefly describe these methods.

Numerical differentiation. The error $\Delta y=\bar{y}-y$ can be expressed as the difference

$$
f\left(\tilde{x}_{1}, \ldots, \tilde{x}_{n}\right)-f\left(x_{1}, \ldots, x_{n}\right)=f\left(\tilde{x}_{1}, \ldots, \tilde{x}_{n}\right)-f\left(\tilde{x}_{1}-\Delta x_{1}, \ldots, \tilde{x}_{n}-\Delta x_{n}\right) .
$$

Usually, the errors $\Delta x_{i}$ are relatively small, so we can neglect the terms that are quadratic in errors. If we neglect quadratic (and higher order terms) in the Taylor expansion of the r.h.s. of (1), we can conclude that

$$
\Delta y=\sum_{i=1}^{n} \frac{\partial f}{\partial x_{i}} \Delta x_{i}
$$

If we know that $\left|\Delta x_{i}\right| \leq \Delta_{i}$, then we can conclude that $|\Delta y| \leq \Delta$, where

$$
\Delta=\sum_{i=1}^{n}\left|\frac{\partial f}{\partial x_{i}}\right| \Delta_{i}
$$

If we know $\sigma\left(x_{i}\right)$, then we can compute $\sigma(y)$ as

$$
\sigma(y)=\sqrt{\sum_{i=1}^{n}\left(\frac{\partial f}{\partial x_{i}}\right)^{2} \sigma^{2}\left(x_{i}\right)} .
$$

Since $f$ is usually given as an algorithm, and not as an analytical expression, we must somehow compute the partial derivatives. We can do that by applying formula (2) to the case when $\Delta x_{i}=h$ for some $i$ and 0 for all other $i$ (here, $h$ is some small number). As a result, we get the following estimate:

$$
\frac{\partial f}{\partial x_{i}}=\frac{f\left(\tilde{x}_{1}, \ldots, \tilde{x}_{i-1}, \tilde{x}_{i}, \tilde{x}_{i+1}, \ldots, \tilde{x}_{n}\right)-f\left(\tilde{x}_{1}, \ldots, \tilde{x}_{i-1}, \tilde{x}_{i}-h, \tilde{x}_{i+1}, \ldots, \tilde{x}_{n}\right)}{h} .
$$

This is a standard formula for numerical differentiation. 
So, in order to get an estimate for $\Delta y$, we must compute the partial derivatives using (5), and then estimate $\Delta$ or $\sigma(y)$ using (3) or (4).

Monte-Carlo methods. A standard Monte-Carlo method (see, e.g., [13]) is used to estimate $\sigma(y)$. This method is based on the following idea: First, we choose the number of iterations $N$. Then, for $k=1, \ldots, N$, we substitute into $f$ the values $\tilde{x}_{i}+\beta_{i}^{k}$, where $\beta_{i}^{k}$ are computersimulated normally distributed random numbers with 0 average and standard deviation $\sigma\left(x_{i}\right)$. As a result, we get $N$ values $y^{1}, \ldots, y^{N}$. Because of (2), the distribution of $y^{k}-y$ is Gaussian, with 0 average and standard deviation $\sigma(y)$. So, we can estimate $\sigma(y)$ as $\sqrt{\sum\left(y^{k}-y\right)^{2} / N}$.

This method gives an approximate value of $\sigma(y)$. In real life, it is sufficient to know $\sigma(y)$ with $\approx 20 \%$ precision (practically no one distinguishes between the cases when the precision is, say, 0.01 , or 0.012 , which is $20 \%$ more). To get such precision, we need $N \approx 25$ iterations.

A Monte-Carlo style method to compute $\Delta$ was proposed in [7] (see also [6]). This method uses $N \approx 50$ iterations to guarantee the desired $20 \%$ precision.

Interval techniques. Standard techniques of interval mathematics require that we dècompose the algorithm $f$ into elementary operations, and then apply interval operations instead of usual ones.

\section{What is wrong with the existing methods}

To use a numerical differentiation method, we must apply the algorithm $f n+1$ times: once to get $\tilde{y}$, and then $n$ times to estimate partial derivatives.

For example, if we have 10 variables, we need 11 times more time to estimate the error than to compute $y$ itself. If $f$ is fast, there is no problem. But if $f$ is already a time-consuming algorithm, this is no good.

For Monte Carlo methods, we must apply $f 25$ or 50 times; so, the computation time for this method is $25-50$ times longer than the time that is necessary to compute $y$.

For interval methods: an operation with intervals consist of 2 or 4 operations with numbers. Therefore, by applying interval mathematics, we increase the computation time at least 2-4 times. In addition, the error estimates that we obtain this way are often "overshoots" (i.e., much bigger than the biggest possible errors).

How to diminish this computation time? If we have several processors that can work in parallel, then we can use parallel algorithms for a speed-up (see, e.g., [6]). But what if we have only one processor?

In this paper, we will describe a frequent real-life situation, when we can drastically decrease this computation time.

\section{The new method of estimating errors}

To develop a new method, we will use the fact that in many real-life situations, the algorithm $f$ is of a very specific type, and for such $f$, we can estimate the error in $y$ really fast.

To elaborate on that, we need to recall where $f$ usually comes from.

Where does $f$ come from? We have mentioned that an algorithm $f$ comes from the known relationship between $x_{i}$ and $y$. Usually, such a relationship exists in a form of a model of the physical phenomenon. By a model, we mean that we have (relatively simple) formulas 
or algorithms that, given some parameters, allow us to compute all physical characteristics, including $x_{i}$ and $y$.

Some of these parameters can be known from the previous experiments (we will denote them by $t_{1}, \ldots, t_{q}$ ). Some of these parameters are specific to this particular situation (we will denote them by $z_{1}, \ldots, z_{p}$ ). These paremeters must be determined from the known measurement results $\tilde{x}_{1}, \ldots, \tilde{x}_{n}$.

So, an algorithm that estimates $\tilde{y}$ from $\tilde{x}_{i}$, consists of two stages:

- first, we use the measurement results $\tilde{x}_{i}$ to get estimates $\tilde{z}_{k}$ for the parameters of the model, and

- second, we use the estimates $\tilde{z}_{k}$ and $\tilde{t}_{l}$ to estimate $y$.

Let us denote by $F_{i}$ and $F$ functions that describe the model, i.e., that express $x_{i}$ and $y$ in terms of $z_{k}$ and $t_{l}: x_{i}=F_{i}\left(z_{1}, \ldots, z_{p}, t_{1}, \ldots, t_{q}\right)$ and $y=F\left(z_{1}, \ldots, z_{p}, t_{1}, \ldots, t_{q}\right)$. Then, to estimate $z_{k}$, we must solve the system of equations $x_{i}=F_{i}\left(z_{1}, \ldots, z_{p}, t_{1}, \ldots, t_{q}\right), 1 \leq i \leq n$. Since we know only approximate values of $x_{i}$, we will get approximate values of $z_{k}$ as well. To improve the precision, we can perform additional measurements. In this case, we will get an over-determined system (with more equations than unknowns). Usually, such systems are solved by the least-squares method, i.e., from the condition that

$$
\sum_{i=1}^{n}\left(F_{i}\left(z_{1}, \ldots, z_{p}, t_{1}, \ldots, t_{q}\right)-x_{i}\right)^{2} \rightarrow \min _{z_{1}, \ldots, z_{p}}
$$

Example. For example, in celestial mechanics, there are rather simple formulas that describe a trajectory of any celestial body in a Solar system (e.g., a comet). The parameters of this trajectory include the position $t_{i}$ of the Sun (that is well known from other measurements), and parameters $z_{k}$ of the orbit relative to the Sun (that have to be determined from the observations). So, if we want to predict a future position $y$ from several observations $\tilde{x}_{i}$, we must find the parameters $z_{k}$ of a trajectory from $\tilde{x}_{i}$, and then predict $y$.

What is the most time-consuming part of this algorithm? If $F_{i}$ are linear, we can easily solve a system of linear equations and find $z_{k}$. The problem becomes computationally complicated when the functions $F_{i}$ are highly non-linear. In this case, no matter what method we use to compute $z_{k}$ (Newton's method, other optimization techniques, etc), all these methods consist of several iterations: we find a first guess $\vec{z}$, substitute these values into $F_{i}$, then adjust the values of $z_{k}$, etc. In other words, the first "back-calculation" stage (estimating $z_{k}$ from $\bar{x}_{i}$ ) includes several "forward" steps (computing $x_{i}$ from $z_{k}$ ). Therefore, the computation time for the first stage is much longer than the time for the second stage.

So, the most time-consuning part of an algorithm $f$ is estimaing $z_{k}$ from $\tilde{x}_{i}$.

Our idea. The reason why existing methods take so long is that they involve several applications of $f$ and, therefore, several repetitions of a time-consuming "back-calculation" step: for the initial values $\overrightarrow{\tilde{x}}=\left(\tilde{x}_{1}, \ldots, \tilde{x}_{n}\right)$, to get the estimates $\tilde{z}_{k}$, and then, for several other input values $\vec{x}$ that are close to $\tilde{x}_{i}$.

Of course, we need to apply this back-calculation once, to get the values $\tilde{z}_{k}$. But next time, when we want to compute $z_{k}$ for some close values $x_{k}$, we do not have to repeat this complicated procedure of solving a system of non-linear equations: indeed, if $x_{i}$ are close to $\bar{x}_{i}$, then the corresponding $z_{k}$ will be close to $\tilde{z}_{k}$. So, it is sufficient to find the small deviations $\Delta z_{k}=z_{k}-\tilde{z}_{k}$. We consider the case when the errors are relatively small, so we can neglect 
the terms that are quadratic in $\Delta z_{k}$, and therefore, instead of the original non-linear system of equations $F_{i}(\vec{z}, \vec{t})=\tilde{x}_{i}$, solve its linearized version.

How to use this idea in case of interval estimates. Since we assume that our model is accurate, the true values $z_{k}$ and $t_{l}$ of the parameters must satisfy the equations $F_{i}\left(z_{1}, \ldots, z_{p}, t_{1}, \ldots, t_{q}\right)=x_{i}$.

If we substitute $z_{k}=\tilde{z}_{k}-\Delta z_{k}, t_{l}=\bar{t}_{l}-\Delta t_{l}$, and $x_{i}=\tilde{x}_{i}-\Delta x_{i}$ into these equations, expand $F_{i}$ into Taylor series, and retain only terms that are linear in $\Delta z_{k}$ and $\Delta t_{l}$, we will conclude that

$$
\sum_{k} a_{i k} \Delta z_{k}=\Delta x_{i}-\delta_{i}-\sum_{l} b_{i l} \Delta t_{l}
$$

where $\delta_{i}=\bar{x}_{i}-F_{i}\left(\tilde{z}_{k}, \bar{t}_{l}\right)$,

$$
a_{i k}=\frac{\partial F_{i}}{\partial z_{k}}, \quad b_{i l}=\frac{\partial F_{i}}{\partial t_{l}} .
$$

From $y=F\left(z_{1}, \ldots, z_{p}, t_{1}, \ldots, t_{q}\right)$, we can likewise conclude that

$$
\Delta y=\sum_{k} c_{k} \Delta z_{k}+\sum_{l} d_{l} \Delta t_{l}
$$

where

$$
c_{k}=\frac{\partial F}{\partial z_{k}}, \quad d_{l}=\frac{\partial F}{\partial t_{l}}
$$

The only thing we know about the values $\Delta x_{i}, \Delta z_{k}$ and $\Delta t_{l}$ is that they satisfy (6) and that the values of $\Delta x_{i}$ and $\Delta t_{l}$ are limited by the corresponsing measurement errors. So, to find possible the interval of possible values of $\Delta y$, we must find the biggest and the smallest value of $\Delta y$ under these conditions. Since these condiditons are linear in the unknowns, this optimization problem becomes a well-known linear programming problem, for whicn fast algorithms are well-known.

So, we are ready to formulate an algorithm.

Algorithm that estimates errors for the case when we know intervals.

Given:

1) An algorithm $F_{i}$ that estimates $x_{i}$ from $z_{k}$ and $t_{l}$.

2) An algorithm $F$ that estimates $y$ from $z_{k}$ and $t_{l}$.

3) An algorithm $f$ that gives an estimate $\tilde{y}$ for $y$ from $\tilde{x}_{i}$ by first estimating $z_{k}$ and then estimating $y$.

4) The measured values $\bar{x}_{i}, 1 \leq i \leq n$ and their precisions $\Delta_{i}$ (i.e., such numbers that $\left.\left|\Delta x_{i}\right|=\left|\bar{x}_{i}-x_{i}\right| \leq \Delta_{i}\right)$.

5) The measured values $t_{1}, \ldots, t_{q}$, and their precisions $\Delta_{l}^{t}$.

Objective: To find an estimate $\Delta$ for $\Delta y=y-\tilde{y}$ (i.e., a value $\Delta$ such that $|\Delta y| \leq \Delta$ ). Algorith:

1) Apply $f$ and get estimates $\tilde{z}_{k}$ and $\tilde{y}$. 
2) Use numerical differentiation to estimate the derivatives (7) and (9).

3) Estimate $\delta_{i}=\Delta x_{i}-F_{i}\left(\tilde{z}_{1}, \ldots, \tilde{z}_{p}, \tilde{t}_{1}, \ldots, \tilde{t}_{q}\right)$.

4) Find $\Delta^{+}$as a solutions of the following linear programming problem with unknowns $\Delta x_{i}, \Delta z_{k}$, and $\Delta t_{l}: \sum c_{k} \Delta z_{k}+d_{l} \Delta t_{l} \rightarrow \max$ under the conditions (6), $-\Delta_{i} \leq \Delta x_{i} \leq \Delta_{i}$ $(1 \leq i \leq n)$, and $-\Delta_{l}^{t} \leq \Delta t \leq \Delta_{l}^{t}(1 \leq l \leq q)$.

5) Find $\Delta^{-}$as a solution to a similar problem, but with min instead of max.

6) Compute $\Delta=\max \left(\Delta^{+},\left|\Delta^{-}\right|\right)$.

Comment. In this algorithm, a time-consuming "back-calculation" part is repeated only once. Therefore, for time-consuming $f$, this algorithm enables us to estimate $\Delta y$ with practiaclly no increase in total computation time.

The case of random errors. In this case, for $x_{i}$ close to $\bar{x}_{i}$, we can also linearize the expression for $F_{i}$. Threfore, instead of a system of non-linear equations, we get a linearized system (6). After this system has been solved, we can use (8) to estimate $\Delta y$.

We must estimate the standard deviation of $y$. Because of (8),

$$
\sigma(y)^{2}=E(\Delta y)^{2}=\sum c_{k} c_{k^{\prime}} E\left(\Delta y_{k} \Delta y_{k^{\prime}}\right)+\sum c_{k} d_{l} E\left(\Delta y_{k} \Delta t_{l}\right)+\sum d_{l} d_{l^{\prime}} E\left(\Delta t_{l} \Delta t_{l^{\prime}}\right) .
$$

So, to estimate $\sigma(y)$, we must estimate these mathematical expetations $E\left(\Delta y_{k} \Delta y_{k^{\prime}}\right)$, etc. If we denote $X_{k}=\Delta z_{k}$ and $X_{p+l}=\Delta t_{l}$, then can say that we must know $E\left(X_{k} X_{l}\right)$ for all $k$ and $l$.

To get these estimates, we can apply the standard methodology of (linear) least squares method (see, e.g., $[8,10]$ ). We are interested in $p+q$ variables $\Delta z_{k}, 1 \leq k \leq p$ and $\Delta t_{l}, 1 \leq l \leq q$. For these unknowns, we have the following system of equations: $\sum a_{i k} \Delta z_{k}+\sum b_{i l} \Delta t_{l} \approx 0$ with standard deviation $\sigma\left(x_{i}\right)$, and $\Delta t_{l} \approx 0$ with standard deviation $\sigma\left(t_{l}\right)$. In terms of $X_{k}$, we have $n+q$ equations: $\sum A_{i k} X_{k} \approx 0$ with standard deviation $\sigma_{i}$, where for $i \leq n: A_{i k}=a_{i k}$ for $k \leq p$, and $A_{i, p+l}=b_{p l}$; for $i>n, A_{n+l, l}=1$ and $A_{n+l, k}=0$ for $k \neq n+l ; \sigma_{i}=\sigma\left(x_{i}\right)$ for $i \leq n$, and $\sigma_{n+l}=\sigma\left(t_{l}\right)$.

According to the least squares theory, the matrix $E\left(X_{k} X_{l}\right)$ is an inverse to the matrix $U_{k l}=\sum_{i} A_{i k} A_{i l} \sigma_{i}^{-2}$. So, we can compute $U_{k l}$, invert it, and use the resulting values of $E\left(X_{k} X_{l}\right)$ to estimate $\sigma(y)$.

Hence, we arrive at the following algorithm:

Algorithm that estimates $\sigma(y)$ when errors $\sigma\left(x_{i}\right)$ are random. Given:

1) An algorithm $F_{i}$ that estimates $x_{i}$ from $z_{k}$ and $t_{l}$.

2) An algorithm $F$ that estimates $y$ from $z_{k}$ and $t_{l}$.

3) An algorithm $f$ that gives an estimate $\tilde{y}$ for $y$ from $\tilde{x}_{i}$ by first estimating $z_{k}$ and then estimating $y$.

4) The measured values $\tilde{x}_{i}, 1 \leq i \leq n$ and their standard deviations $\sigma\left(x_{i}\right)$.

5) The measured values $t_{1}, \ldots, t_{q}$, and their standard deviations $\sigma\left(t_{l}\right)$.

Obective: To find a standard deviation $\sigma(y)$.

Algorithm:

1) Apply $f$ and get estimates $\tilde{z}_{k}$ and $\tilde{y}$. 
2) Use numerical differentiation to estimate the derivatives (7) and (9).

3) Compute $A_{i k}, \sigma_{i}$, and $U_{k l}$ (as above).

4) Invert the matrix $U_{k l}$ and get $E\left(X_{k} X_{l}\right)$.

5) Substitute the resulting values of $E\left(X_{k} X_{l}\right)$ into the formula (10) and thus compute $\sigma(y)$.

Comments.

1. In this algorithm, a time-consuming "back-calculation" part is also repeated only once. Therefore, for time-consuming $f$, we can estimate $\tilde{y}$ and $\sigma(y)$ with practically no increase in total computation time.

2. If some parameters $t_{l}$ are already known with much better precision than our measurements, then we can simplify our computations by asuming that they are absolutely precise $\left(\Delta t_{l}=0\right)$. In this case, it is sufficient to consider fewer than $p+q$ variables, and thus, the computation will be even smaller. Another case when we can invert matrices of sizes smaller than $(p+q) \times(p+q)$ is when some of the parameters $t_{l}$ do not influence $x_{i}$ at all, and are used only to compute $y$. For such parameters $t_{l}, E\left(\left(\Delta t_{l}\right)^{2}\right)=\sigma\left(t_{l}\right)^{2}$, and $E\left(\Delta t_{l} \Delta z_{k}\right)=0$ for all $k$.

\section{Case study: pavement engineering}

The problem. One of the main problems of pavement engineering is to predict the remaining lifetime $y$ of a pavement. It is impossible to measure $y$ directly, so instead a Falling Weight Deflectometer (FWD) is used: we drop a mass on the pavement, and measure the resulting deviations $x_{1}, \ldots, x_{n}$ in several (usually $n=7$ ) locations on the surface of the pavement.

To estimate $y$ from $\bar{x}_{i}$, a 3 -layer pavement model is used $[9,16]$. According to this model, the pavement consists of 3 layers: 1st asphaltic/concrete, 2nd base, 3rd subgrade layer. Each layer is characterized by 3 paremeters: its thickness $h_{i}$, its Poisson ratio $\nu_{i}$, and its elastic modulus $E_{i}$.

Thickness $h_{i}$ does not change in time, so we can take $h_{i}$ from the documents that described the design of this pavement. The values $\nu_{i}$ may somewhat change. However, $\nu_{i}$ describe the horizontal strain, and the pavement is designed for vertical loads only. So, these changes do not influence the lifetime, and we can neglect them.

So, in this problem, we have 7 parameters $t_{l}$ whose values are known from the previous measurements: $h_{1}, h_{2}, h_{3}, \nu_{1}, \nu_{2}, \nu_{3}$, and the weight $w$ of the FWD mass. We have 3 parameters $z_{k}$ that have to be determined from the measurements: $E_{1}, E_{2}, E_{3}$. Corresponding algorithms $F_{i}$ and $F$ are given in $[9,16]$ (here, $F$ is also a two-stage algorithm: given $z_{k}$ and $t_{l}$, we first compute tensile and compressive strains $\varepsilon_{t}$ and $\varepsilon_{v}$, and then use these strains to predict $y$ ).

How errors were estimated before. In this problem, all the sensors are calibrated, so we can assume that the errors are random. In $[12,17]$, a Monte-Carlo method was used.

Computation time of the existing method. On a 386 IBM PC, forward computations (i.e., computing $y$ and $x_{i}$ from the known parameters $z_{k}$ and $t_{l}$ ) take about 20 seconds. A program $f$ that estimates $y$ from $\tilde{x}_{1}, \ldots, \tilde{x}_{n}$ takes about 3 minutes to run. The Monte-Carlo method required up to 25 repetitions of this program, so its total computation time is about $80 \mathrm{~min}$. If we take into consideration that we must measure the value of $y$ for every mile, it is too long. 
Our results. We applied the above algorithm to estimate $\sigma(y)$ (for details, see [3]). The entire algorithm, including both computing $\tilde{y}$ from $\bar{x}_{i}$, and estimating $\sigma(y)$, ran for about 3.5 minutes. This time is close to the time $(3 \mathrm{~min})$ required to compute $y$ from $\bar{x}_{i}$.

\section{Conclusion}

With practically no increase in the computation time, we not only compute $y$, but we also estimate its precision.

\section{References}

[1] Beck, J. V. Parameter estimation in engineering and science. Wiley, 1977.

[2] Breipohl, A. M. Probabilistic systems analysis. Wiley, 1970.

[3] Chang, C.-C. Fast algorithm that estimates the precision of indirect measurements. Master Project, Computer Science Dept., University of Texas at El Paso, 1992.

[4] Fuller, W. A. Measurement error models. Wiley, 1987.

[5] Handbook of engineering mathematics. American Society for Metals, 1983.

[6] Kreinovich, V., Bernat, A., et al. Parallel computers estimate errors caused by imprecise data. Interval Computations 1 (2) (1991), pp. 31-46.

[7] Kreinovich, V. and Pavlovich, M. I. Error estimate of the result of indirect measurements by using a calculational experiment. Measurement Techniques 28 (3) (1985), pp. 201-205.

[8] Linnik, Yu. V. Method of least squares and principles of the theory of observations. Pergamon Press, 1961.

[9] Lytton, R. L. Backcalculation of pavement layer properties. In: Bush III, A. J. and Baladi, G. Y. (eds) "Nondestructive Testing of Pavements and Backcalculation of Moduli, ASTM STP 1026", American Society for Testing and Materials, Philadelphia, 1986, pp. 7-38.

[10] Mikhail, E. M. Observations and least squares. Thomas Y. Crowell, 1976.

[11] Moore, R. E. Methods and applications of interval analysis. SIAM, Philadelphia, 1979.

[12] Rodriguez-Gomez, J., Ferregut, C., and Nazarian, S. Impact of variability in pazement parameters on backcalculated moduli. University of Texas at El Paso, Dept. of Civil Engineering, Techn. Rep., 1991.

[13] Rubinstein, R. Y. Simulation and the Monte Carlo method. Wiley, 1981

[14] Scheaffer, R. L. and McClave, J. T. Probability and siatistics for engineers. PWS-KENT, 1986.

[15] Sneddon. I. N. Encyclopaedic dictionary of mathematics for engineers and applied scientists. Pergamon Press, 1976.

[16] Uzan, J. et al. A microcomputer based procedure for backcalculating layer moduli from FWD data. Texas Transportation Institute Research Report 1123-1, 1988. 
[17] Vennalaganti, K. M., Ferregut, C., and Nazarian, S. Stochastic analysis of errors in remaining life due to misestimation of pavement parameters in NDT. University fo Texas at El Paso, Civil Engineering Dept., Techn. Rep., 1992.

Received: March 10, 1993

C. Ferregut, S. Nazarian, K. Vennalagant Department of Civil Engineering University of Texas at El Paso El Paso, TX 79968

USA

C.-C.Chang, V. Kreinovich Computer Science Department University of Texas at El Paso El Paso, TX 79968 USA

C.-C.Chang, current address: 8 Kan Lo St., Taichung, Taiwan Republic of China 